\title{
Experimental Investigation of Innovative Internal Trailing Edge Cooling Configurations with Pentagonal Arrangement and Elliptic Pin Fin
}

\author{
L. Tarchi, ${ }^{1}$ B. Facchini, ${ }^{1}$ and S. Zecchi ${ }^{2}$ \\ ${ }^{1}$ Dipartimento di Energetica "Sergio Stecco", Università di Firenze, Via S. Marta 3, 50139 Firenze, Italy \\ ${ }^{2}$ AVIO S.P.A, Via I Maggio 99, 10040 Rivalta di Torino, Italy
}

Correspondence should be addressed to L. Tarchi, lorenzo.tarchi@htc.de.unifi.it

Received 6 March 2008; Accepted 6 August 2008

Recommended by Ken-Ichi Funazaki

This paper describes a heat transfer experimental study of four different internal trailing edge cooling configurations based on pin fin schemes. The aim of the study is the comparison between innovative configurations and standard ones. So, a circular pin fin configuration with an innovative pentagonal scheme is compared to a standard staggered scheme, while two elliptic pin fin configurations are compared to each other turning the ellipse from the streamwise to the spanwise direction. For each configuration, heat transfer and pressure loss measurements were made keeping the Mach number fixed at 0.3 and varying the Reynolds number from 9000 to 27000. In order to investigate the overall behavior of both endwall and pedestals, heat transfer measurements are performed using a combined transient technique. Over the endwall surface, the classic transient technique with thermochromic liquid crystals allows the measurement of a detailed heat transfer coefficient (HTC) map. Pin fins are made of high thermal conductivity material, and an inverse data reduction method based on a finite element code allows to evaluate the mean HTC of each pin fin. Results show that the pentagonal arrangement generates a nonuniform HTC distribution over the endwall surface, while, in terms of average values, it is equivalent to the staggered configuration. On the contrary, the HTC map of the two elliptic configurations is similar, but the spanwise arrangement generates higher heat transfer coefficients and pressure losses.

Copyright (C) 2008 L. Tarchi et al. This is an open access article distributed under the Creative Commons Attribution License, which permits unrestricted use, distribution, and reproduction in any medium, provided the original work is properly cited.

\section{INTRODUCTION}

The trailing edge is one of the most critical parts of gas turbine blades and vanes since it is exposed to very high thermal loads. A very efficient cooling system is, therefore, required so as to keep metal temperature below critical values. Inline and staggered arrays of short cylindrical pin fins are one of the most common types of cooling devices used in turbine blades. Such arrays enhance the heat transfer levels mainly increasing the heat transfer coefficient and, for $H / D>0.5$, the wet surface. Being easier to manufacture, pin fins with circular cross-sections are the most used and investigated. The first experimental investigations on circular pin fins were carried out by [1-3]. They measured row by row heat transfer coefficients and pressure losses for inline and staggered configurations. Reference [4] investigated the influence of accelerating flow in a wedge-shaped duct on heat transfer. Their results showed that flow acceleration decreases the influence of Reynolds number on Nusselt number. By means of the transient TLC technique, $[5,6]$ studied the effects of the fillet radii on the endwall heat transfer, while [7-9] studied the effects of turning flow in a wedge-shaped duct with circular, elliptical, and diamond cross-section pin fins. It has been demonstrated by various authors $[23,24]$ that cylinders with streamline-shaped crosssection have much less flow resistance than circular ones, while they have about the same behavior in terms of heat transfer. The work in [10] also investigated the partial length circular pin fin concept and found that both the array averaged-heat transfer and friction factor decrease with increasing gap distance.

Even if streamwise-oriented elliptic pin fins show an overall better behavior than other shapes, devices with a non-aerodynamic shape are employed in gas turbine airfoils too. In modern multipass cooling systems, the airflow approaches the trailing edge region from the hub or from 


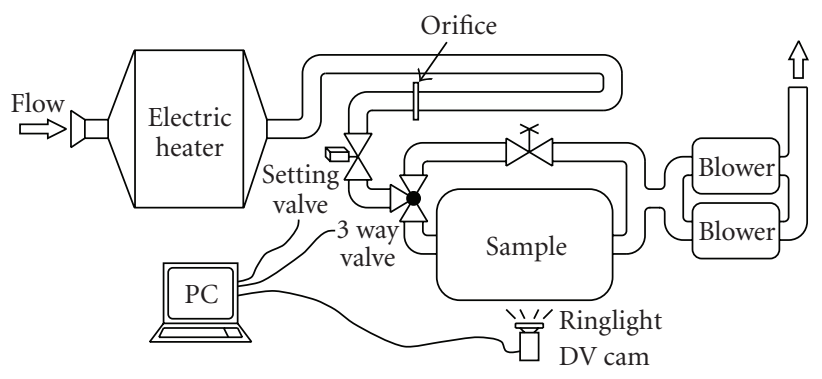

Figure 1: Experimental setup.

the tip of the airfoil; hence, the implementation of cooling devices with low pressure losses could lead to nonuniform coolant distribution in the radial direction and then to higher differences in airfoil temperature. That is the reason why cooling devices with high pressure losses have been implemented and investigated. Reference [8] studied the effects of diamond pin fins and turning flow on heat transfer. Pin fins with oblong cross-section were investigated by [11] for various pin orientations with respect to the main flow. Their results indicate that the use of elongated pin fins (oblong shape) increases endwall heat transfer and also causes higher levels of aerodynamic penalty than the circular pin fins when the main flow direction deviates from the direction of the major axis of the oblong pin fin. When the main flow approaches zero incidence, the pressure loss levels become lower than circular pin fins ones.

In the present paper, the differences between streamwiseand spanwise-oriented elliptic cross-section pin fins are investigated. Pin fins are inserted in a wedge-shaped duct in order to replicate a typical trailing edge cooling system. Then, two geometries with circular cross-section are investigated: one is a standard staggered array, while the other consists of an innovative array based on a pentagonal scheme. As already mentioned, in modern cooling systems, the flow does not approach the pin fin array in the axial direction, but in a mixed axial-radial direction. In this case, a staggered pin fin array works as an in line configuration, leading to lower heat transfer capability. Hence, the idea is to develop an innovative array insensitive to the mainstream direction. The aim of this paper is then the comparison in terms of heat transfer and pressure losses between the standard staggered array and the innovative pentagonal scheme, with a mainstream flow oriented in the axial direction. An experimental survey with mixed axial-radial flow is planned as well.

About the experimental techniques, in the pioneering works of $[1,3]$, an average HTC row by row value was evaluated employing copper test articles and a steadystate technique. Afterwards, $[12,13]$ used the naphthalene sublimation method, based on heat-mass transfer analogy, to investigate the separate contribution of endwall and pin fin. The work in $[5,6]$ performed detailed heat transfer measurements on the endwall surface of pedestals array with TLC transient technique. With the same experimental method, $[7,9,14]$ evaluated heat transfer and pressure losses in trailing edge cooling geometries typical of real blades: wedge and trapezoidal ducts, pin shape, and lateral flow effects were investigated. Lately, [15], besides the endwall HTC measurements with TLC, evaluated the pin fin contribution to heat transfer using high conductivity pedestals and an inverse data reduction method based on a finite element simulation of the transient test. Results shown that pin fins have higher HTC than the surrounding endwall surface. On the contrary, [16], using the so-called "lumped heat capacity method" to estimate the pin fin contribution, measured lower heat transfer values over the pin fin surface.

\section{EXPERIMENTS}

\subsection{Test facility}

The experimental survey was performed at the Dipartimento di Energetica of the University of Florence. The final aim of this activity is the measurement of the HTC over the whole internal surfaces of four different geometries using a transient technique.

The test rig (Figure 1) consists of a suction-type circuit that allows complete control of the air stream in terms of both temperature and mass flow rate. The mainstream air passes through a $9.0 \mathrm{~kW}$ electronically controlled electric heater; then, the flow rate is measured by an orifice. A three-way valve, with pneumatic actuator, assures the sample at room temperature, as required by transient technique, while the other components of the test rig are warming up. Two rotary vane vacuum pumps, powered by two $7.5 \mathrm{~kW}$ electric motors, blow air outside and provide the suction for a maximum mass flow rate of $0.10 \mathrm{~kg} / \mathrm{s}$. The flow rate is set up by guiding the motor speed between $300 \mathrm{rpm}$ and $1300 \mathrm{rpm}$ and by throttling the remote-controlledmotorized valve; the air temperature exiting the heater is controlled by means of a four-wire RTD (Pt100). Two pressure scanners Scanivalve DSA 3017 with temperaturecompensated piezoresistive relative pressure sensors allow us to measure the total or static pressure in 32 different locations with an accuracy of 6.9 Pa. Several T-type thermocouples connected to a data acquisition/switch unit (HP-Agilent 34970A) measure the mainstream temperature and the aluminum pin fin temperature. A digital three-charge-coupleddevice (3CCD) camcorder (Canon XM-2) records a sequence of color bitmap images $(720 \times 576$ pixel, 25 frames $/ \mathrm{s})$ from the thermochromic liquid crystal- (TLC-) painted surface on a PC (IEEE-1394 standard). The illuminating system (ShottFostec KL1500 LCD) uses an optical fiber ring light to ensure a uniform illumination on the test surface, and it allows us to keep both color temperature and light power constant. In order to reduce any undesired polymethyl methacrylate (PMMA) reflections, two polarized lens filters are fitted on both ring light and camcorder lenses. TLCs are the devices used to evaluate surface temperature and, consequently, the heat transfer coefficient. For our purpose, we used the $40 \mathrm{C} 5 \mathrm{~W}$ formulation of Hallcrest active from $40^{\circ} \mathrm{C}$ to $45^{\circ} \mathrm{C}$. Crystals are thinned with water and sprayed with an airbrush on the test surface, then a black background paint 


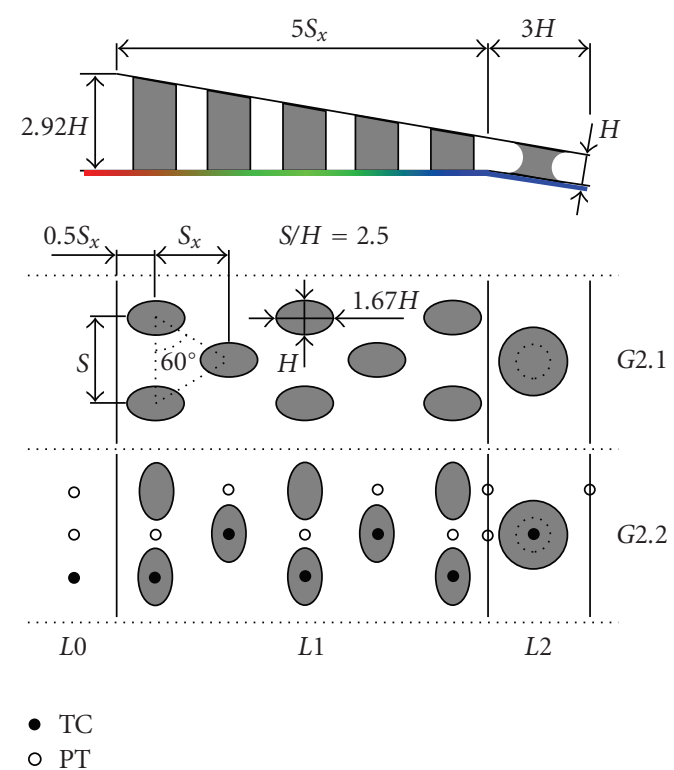

FIgURE 2: Streamwise and spanwise staggered elliptical pin fin configurations.

is applied. TLCs have been calibrated, replicating the same optic conditions of the real test: the peak value of the green intensity was found at $42.3^{\circ} \mathrm{C}$, so it has been used in the data reduction procedure.

\subsection{Geometries}

Four different pin fin configurations are investigated in this paper. In Figure 2, a sketch of the two elliptic configurations is shown. In the first one (G2.1), the major axis of the ellipse is oriented in the airflow direction (i.e., streamwise), while in the other configuration (G2.2), the ellipse is rotated 90 degrees, so in spanwise direction. Both the arrays are fitted in a 10-degree wedge-shaped duct ( $L 1$ region), replicating the typical trailing edge shape. Ahead of that region, the test article starts with a settling chamber, a grid, and then a smooth constant height duct ( $L 0$ region). $L 1$ region is $200 \mathrm{~mm}$ wide and $5 S_{x}=72.75 \mathrm{~mm}$ long. Height varies from $H_{L 0}=19.65 \mathrm{~mm}$ to $H_{L 2}=H=6.72 \mathrm{~mm}$. Each pin fin row is composed of 12 or 11 pin fins with diameter $D=$ $H$. Spanwise pitch is $S / D=2.5$ and the array is made by equilateral triangles, so $S_{x} / D=2.17$. The $L 2$ region consists of a constant height duct with a single row of circular pin fin with fillet radius $r=H / 2$ and the minimum diameter being equal to the $L 1$ pins diameter. The fillet radius was introduced because it represents with more accuracy a typical configuration used in the outlet of trailing edge cooling systems of high-pressure stages.

The two other configurations are composed by circular pin fins (Figure 3). The geometry G2.5 consists of 7 rows of staggered pin fins with diameter $D=H=5.6 \mathrm{~mm}$, spanwise pitch $S / D=2.3$ and streamwise pitch $S_{x} / D=1.86$. Endwall dimensions are the same of the elliptic geometries while the height is different $\left(H_{L 0}=18.51 \mathrm{~mm}-H_{L 2}=H=5.6 \mathrm{~mm}\right)$. Each row presents 15 pins, thus over the whole $L 1$ region,

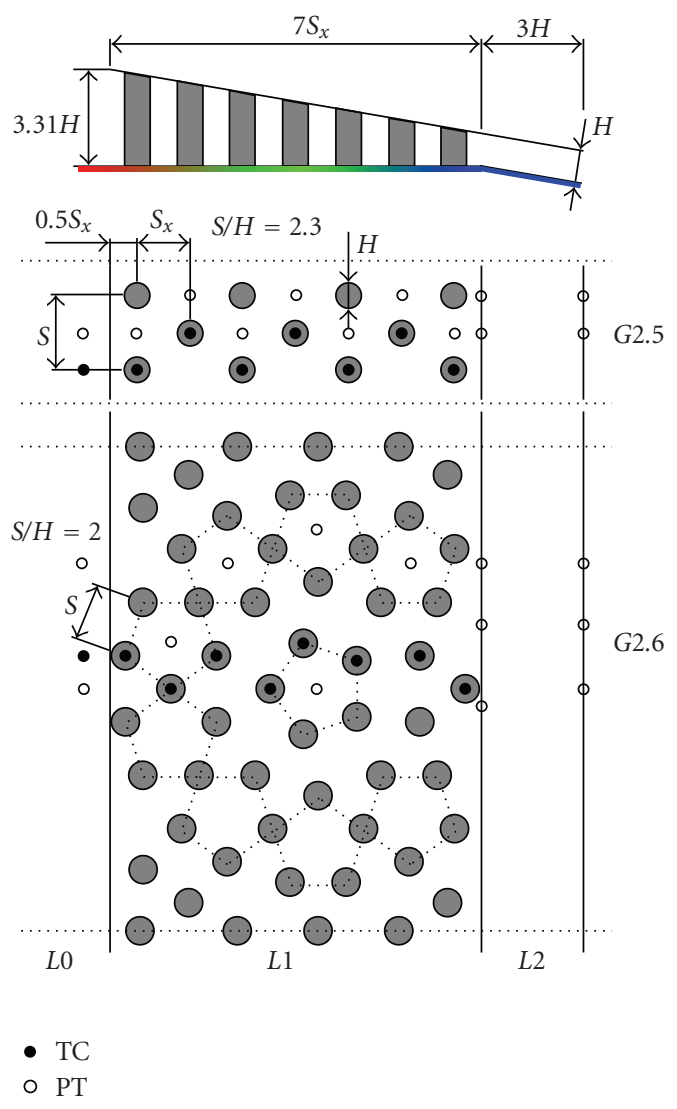

Figure 3: Circular pin fin geometries with staggered (G2.5) and pentagonal arrangement (G2.6).

there are 105 pedestals. In the G2.6 configuration, there are 106 pin fins arranged in a pentagonal scheme. The design of such innovative geometry starts from the idea to develop a repeatable pin fin array capable of good heat transfer performance in presence of mixed axial-radial coolant flows as well. As a matter of fact, the standard staggered array works very well once the airflow is orthogonal to the array, while in presence of inclined airflow, the array works as an inline configuration, and cooling performance decreases. Results reported in this paper intend to verify the behavior of the pentagonal scheme considering an axial coolant flow in comparison with the standard staggered scheme, keeping practically constant the overall pin fin density ratio (105 or 106 pin fins over the $L 1$ region). In the $L 2$ region, there are no pin fins because G2.5 and G2.6 configurations represent a real cooling system where along the $L 2$ region a cutback is present.

As required by the transient technique, models are made of transparent PMMA, and the TLC were applied over the whole endwall from $L 0$ to $L 2$. On the contrary, pin fins are made of aluminum and their temperature was measured with a small thermocouple inside one pin for each row. Air temperature is also measured at the inlet with two miniaturized thermocouples that allow a fast response for the transient test. The inlet temperature profile was verified during the commissioning of the test article and it is uniform in 
the whole test section. As regarding pressure measurements, static pressure is measured in various sections from $L 0$ to L2. In Figures 2 and 3, the position of thermocouples and pressure taps for each model is depicted with white and black circles (G2.1 instrumentation is exactly in the same position of G2.2 geometry).

\subsection{Experimental procedure}

Heat transfer tests were performed using a combined transient technique that allows the measurement of HTC on both endwall and pin fin surfaces with a single transient test. During the warm up of the rig, the test model is kept at constant temperature. When air temperature reaches about $70-80^{\circ} \mathrm{C}$ in the bypass circuit, the 3 -way valve is switched making the air passing through the test model; automatically air temperature, aluminum pin fin temperature, and air pressure values are recorded and the camcorder starts acquiring frames of the TLC-coated surface. The transient test is finished when the liquid crystal reaches the blue color over the whole surface.

Pressure losses are evaluated with a cold test.

\section{DATA REDUCTION}

\subsection{Reynolds and Nusselt numbers definition}

Reynolds and Nusselt numbers for data reduction are defined in two different ways: the first is based on the inlet section (LO) hydraulic diameter, the second on the pin fin diameter $D$. In both the elliptic configurations, the minor axis length is used as reference diameter:

$$
\begin{aligned}
\operatorname{Re}_{L 0} & =\frac{\dot{m} D_{L 0}}{A_{L 0} \mu}, & \mathrm{Nu}_{L 0} & =\frac{\bar{h} D_{L 0}}{k}, \\
\operatorname{Re}_{d} & =\frac{\dot{m} D}{A_{\min } \mu}, & \mathrm{Nu}_{d} & =\frac{\bar{h} D}{k} .
\end{aligned}
$$

$D_{L 0}$ is the hydraulic diameter of the inlet duct with crosssection area $A_{L 0} ; \mu$ and $k$ are evaluated at the total temperature measured in the $L 0$ region; and $A_{\min }$ is the minimum passage area between two pins and it is variable row by row. In order to compare directly the two elliptic configurations, the minimum passage area of the streamwise configuration (G2.1) is used in the data reduction of the spanwise (G2.2) too. Similarly, the pentagonal geometry (G2.6) results were postprocessed using the minimum passage area of the staggered configuration (G2.5).

The overall average HTC $\bar{h}$ is defined as follows:

$$
\bar{h}=\sum_{i=1}^{N} \frac{h_{\mathrm{EW} i} \cdot A_{\mathrm{EW} i}+h_{\mathrm{PIN} i} \cdot A_{\mathrm{PIN} i}}{A_{\mathrm{EW} i}+A_{\mathrm{PIN} i}},
$$

where $A_{\mathrm{EW}}$ takes into account the lower and the upper endwall surfaces. In the definition of the row by row Nusselt number (2), the average HTC is based on single-row data.

\subsection{Heat transfer coefficient evaluation}

Detailed heat transfer coefficient distribution on the endwall surface is obtained assuming one-dimensional conduction over a semi-infinite solid $[17,18]$. The "series of steps" method [19] is used to take into account the air temperature time history.

Because of the high heat transfer coefficients achieved during the test and the quite high wet surface, the mainstream temperature decreases between the inlet and the outlet section is not negligible; thus the use of the inlet temperature measured in $L 0$ as reference temperature leads to underestimate the HTC. Solving such a problem makes necessary to take into account the variation of the local bulk mean temperature in time and space. Reference [20] evaluate four different approaches and their theoretical background for determining the local bulk mean temperature and the sensible local heat transfer coefficient. These authors assert that the invariant local heat flux method is the best choice as it produces very accurate results, with a very little processing time and implementation effort; so, such method was applied in the data reduction procedure.

Pin fin heat transfer coefficients are evaluated by means of an inverse data reduction method. Such method is based on a full 3D transient FEM simulation of the experiment and an iterative procedure. The HTC of each pin fin is updated using the Newton convergence criterion, iterating until the temperature history evaluated with the FEM code matches the measured temperature history inside each pin. A moredetailed description of this procedure was reported by [15].

\subsection{Pressure drops evaluation}

Pressure drops were measured across the duct in adiabatic conditions (mainstream flow at ambient temperature). Static pressure was measured in various points, starting from the inlet, until the end of the $L 2$ region (Figures 2 and 3 ). The pressure values at the end of each region were used to evaluate the friction factor defined as

$$
f=\frac{\Delta p_{0}}{(1 / 2) \rho v^{2}},
$$

where $\Delta p_{0}$ is the total pressure difference between the beginning and the end of the $L 1$ region; the total pressure is calculated summing up the dynamic pressure to the average static pressure of each section. $\rho$ and $v$ are average values measured in the $L 0$ region.

\subsection{Experimental uncertainty}

The uncertainty analysis was performed following the standard PTC 19.1 [21] based on the Kline and McClintock method [22]. Typical uncertainties of the most important parameters are HTC $=12.2 \%, \operatorname{Re}=2.8 \%, f=$ $5.4 \%$. More details about the individual contributions to the uncertainties of the single parameters for each of the measured physical properties are reported by [15].

\section{RESULTS}

\subsection{Elliptic pin fin configurations}

For each configuration, five tests were performed at different Reynolds numbers $\left(9000<\operatorname{Re}_{d 5}<27000\right)$, keeping constant 


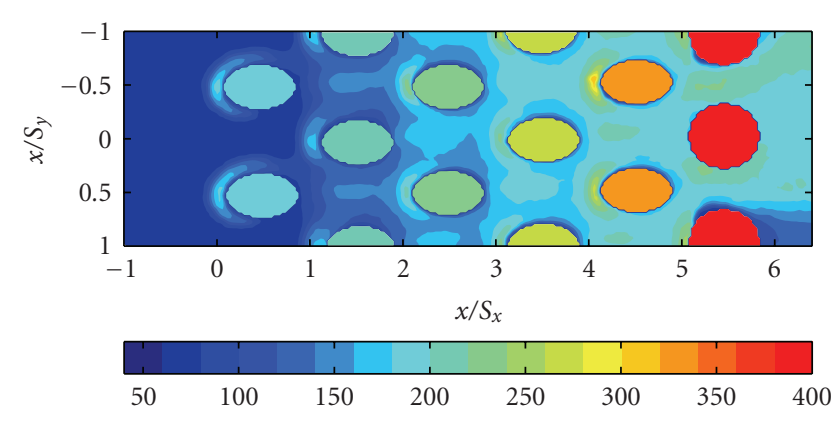

(a)

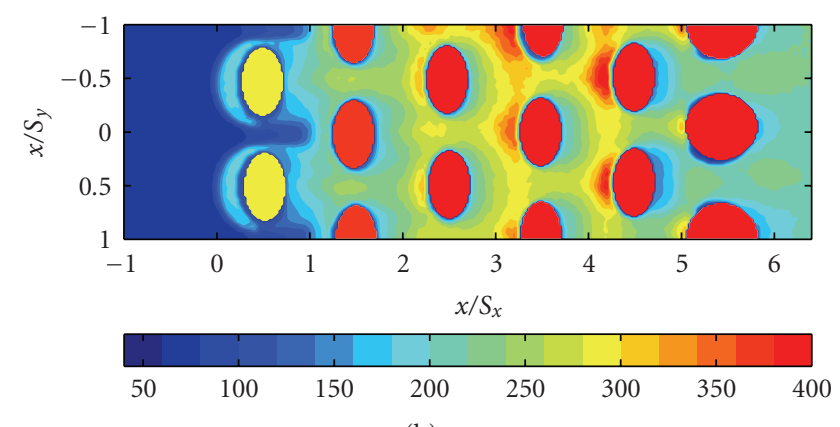

(b)

FIGURE 4: HTC $\left[\mathrm{W} / \mathrm{m}^{2} \mathrm{~K}\right] \mathrm{map}$ of elliptic configurations- $\mathrm{Re}_{d 5}=$ 18000 .

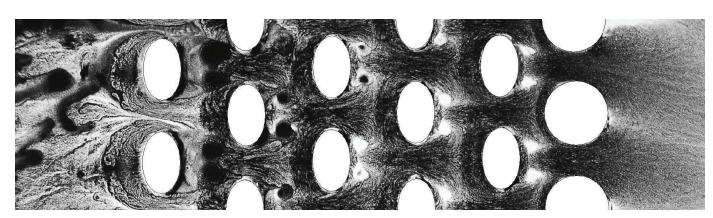

Figure 5: Surface flow visualization of G2.2.

the Mach number at 0.3 . Both $\mathrm{Ma}$ and $\mathrm{Re}_{d}$ are evaluated in the minimum passage area between two pins of the fifth row (i.e., in the throat section). Figure 4 shows a detailed map of the heat transfer coefficient of the two elliptic configurations at $\operatorname{Re}_{d 5}=18000$. The colors inside the pins correspond to the average HTC measured over the pin fin surface with the inverse data reduction. As the local HTC peak values show, a stagnation area is present in both configurations ahead of each pin fin, while the recirculation zone presents slight differences especially in the first row: in G2.1, the recirculation area is small and does not lead to a large increase of HTC, whereas, in G2.2, the larger recirculation generated by the wake of the first pin enhances the heat transfer. For a deeper insight into the surface flow structure, a surface flow visualization of this configuration was done using the oil and dye technique. The endwall flow pattern in Figure 5 shows a large stagnation region ahead of the first pin, with the saddle point located at $1 D$ upstream the pin. The two counter-rotating vortexes in the recirculation downstream the first pin cover a quite large area and they spread up to second row, interacting with the stagnation region of such row that is not present on the endwall. As from the following rows, the flow pattern becomes more periodic,

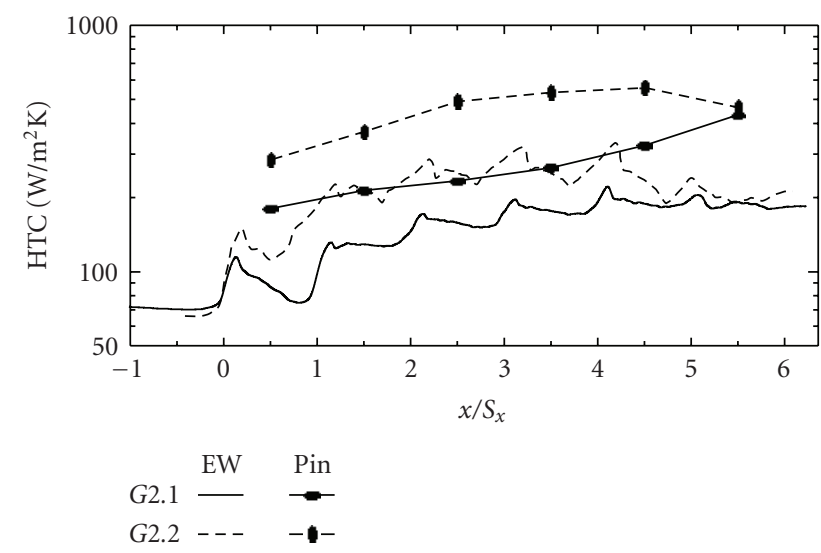

Figure 6: Endwall spanwise averaged and pin fin $\mathrm{HTC}-\mathrm{Re}_{d 5}=$ 18000 .

the recirculation is smaller, and the saddle point is always located at about $D / 2$ upstream the pin leading edge.

Figure 6 reports the spanwise-averaged values of HTC together with the pin fin surface average values. First of all, it is evident how the spanwise-oriented pin fins (G2.2) generate a more turbulent flow and then higher heat transfer rates over the whole endwall. Moreover, the increase in streamwise direction due to the combined effect of pin fin and convergence is clearly visible for both configurations in the $L 1$ region $\left(0<x / S_{x}<5\right)$, while endwall values are quite constant over the $L 2$ region $\left(x / S_{x}>5\right)$. About this region, we have to point out that HTC values are very similar for both configuration, showing that the high turbulence generated by the streamwise pin fins quickly vanishes.

A final important issue to be discussed concerns with the different contribution to heat transfer of pin fins and endwall. Looking at Figures 4 and 6, it is noticeable that pin fin HTC is always higher than the surrounding endwall one, and always very close to the peak value located upstream each pin. For the first row pin fin, HTC are about twice than the endwall ones; according to the authors, this trend can be explained thinking over the flow field of such region. Only a portion of the endwall is covered by the horseshoe vortex generated by the pin, while between the pins, specially in the streamwise configuration (G2.1), there are some areas with the same HTC of the upstream flow. On the contrary, the pin fin surface is fully covered by flow structures with high heat transfer: a stagnation region over the leading edge and a recirculation over the back side. In the following rows, the differences between pin fin HTC and endwall is slightly lower as the first are $50-90 \%$ higher than the latter. This behavior was reported by various researchers that investigated the separate contribution to heat transfer of pin fin and endwall. Reference [3] reported that, for a staggered array, the HTC on the pin surface is $35 \%$ higher than the endwall values. Reference [11] found that the ratio $h_{\mathrm{PIN}} / h_{\mathrm{EW}}$ varies from 1.8 to 2.1 , depending on steamwise pitch $\left(S_{x}\right)$. Reference [13], using the naphthalane sublimation technique, measured that HTC over the pin fin surfece is $10-20 \%$ higher than the endwall values. Finally, [15], using the same combined data 


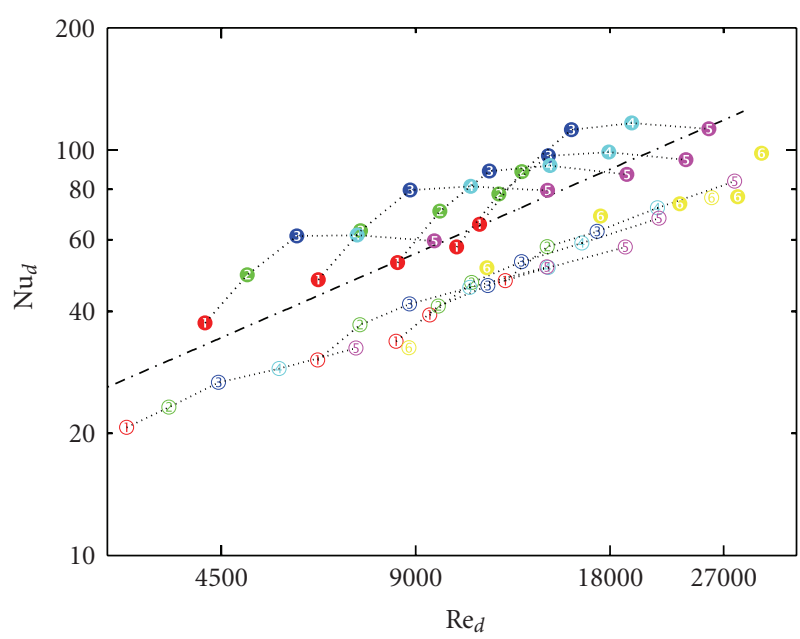

Pin 1 Pin 2 Pin 3 Pin 4 Pin 5 Pin 6

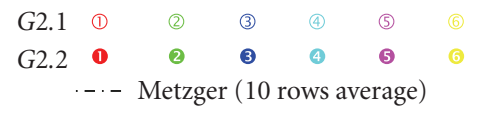

Figure 7: Row by row heat transfer data.

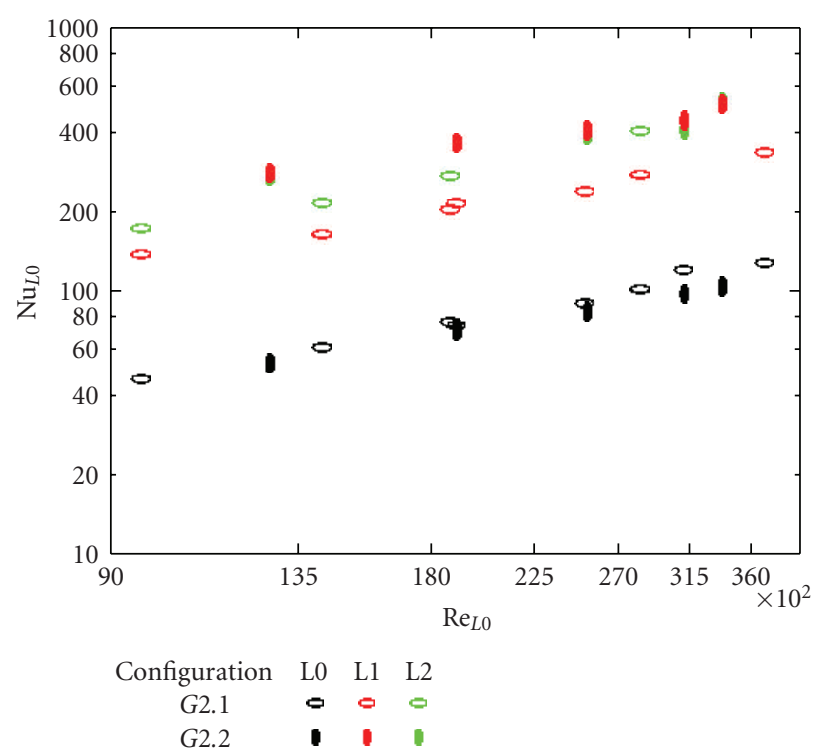

Figure 8: G2.1 and G2.2 data- $\mathrm{Nu}_{L 0}$ versus $\mathrm{Re}_{L 0}$.

reduction method reported in this paper, showed that the pin HTC is always higher than the endwall. Recently, [16], using a "lumped heat capacity method" for the pin fins, reported an inverse result; they mesured a higher HTC over the endwall by about $3-40 \%$ than that on the corresponding pin fin of the same row.

Figures 7 and 8 present all the experimental data in the two different definitions of Reynolds and Nusselt number reported in (1) and (2).

The general trend of the experimental data confirms the results of the already discussed $\operatorname{Re}_{d 5}=18000$ test, so the spanwise configuration (G2.2) reveals higher heat transfer values at the same mass flow rate. Moreover, data scattering of such configuration is higher; such behavior is mainly due to the steep increase of heat transfer capability between the first and the third rows, while values are quite constant between the third and the fifth rows .

Such trend is present in the G2.1 configuration too, even if it is hardly visible. Reference [4] in a 10 row-staggered pin fin configuration with constant height found that the average heat transfer increases up to the 4 th-5th row, then decreases up to the 10th row. In the present results, the general trend is the same measured by [4], but taking also into account the row by row increasing $\mathrm{Re}_{d}$, the local maximum is reached at the 3rd row and is much more enhanced in the spanwiseoriented geometry.

L2 region values (PIN6) of both configurations are in line with the G2.1 data, showing that the very high turbulence levels generated by the spanwise-oriented elliptic fins decrease very quickly without affecting the heat transfer behavior of the $L 2$ region.

The comparison with the correlation proposed by [4] (Figure 7) for a 10-row-staggered array with $1.5<S_{x} / D<$ $5.0, S / D<2.5$ and $H / D=1.0$ shows the effect of the elliptic pin fins on heat transfer. This correlation showed also a good agreement with a circular pin fin array inserted in a wedge-shape duct similar to the present work [15]. Looking at the graph, it is clearly visible that the streamwise-oriented pin fins experience lower heat transfer coefficients than the circular ones. Such result, as also described by other authors $[23,24]$, is mainly due to the different wake behavior of the two devices: while circular pin fins produce a wake with two large counter-rotating vortexes, for the elliptic pin fin configurations, such vortexes usually are not present. Hence, streamwise elliptic pin fins produce less turbulence and then lower HTC values. On the contrary, in the spanwise-oriented configuration, the wakes cover a large part of the endwall surface (see Figure 5), and then the heat transfer is highly enhanced.

In order to have a general overview about the cooling performance of the two elliptic geometries, Figure 8 shows the experimental data of the whole $L 1$ region, together with $L 0$ and $L 2$ values. As expected, the entrance region $(L 0)$ is not affected by the pin fin orientation, while in the $L 1$ region, the higher Nusselt number values of the spanwise configuration are evident.

As already highlighted in the row by row data reduction (Figure 7), in the constant height region (L2) with circular filleted pin fins, the elliptic pin orientation does not have a large effect on the heat transfer behavior.

\subsection{Staggered and pentagonal scheme configurations}

For each configuration, five tests were performed at different Reynolds numbers $\left(9000<\operatorname{Re}_{d 7}<27000\right)$, once again keeping the Mach number at 0.3. Both $\mathrm{Ma}$ and $\mathrm{Re}_{d}$ evaluated in the minimum passage are between two pins of the seventh row (i.e., in the throat section). Figure 9 depicts a detailed map of the heat transfer coefficient of the standard staggered configuration and of the innovative configuration with pentagonal arrangement at $\operatorname{Re}_{d 7}=18000$. The colors inside the pins correspond to the average HTC measured 


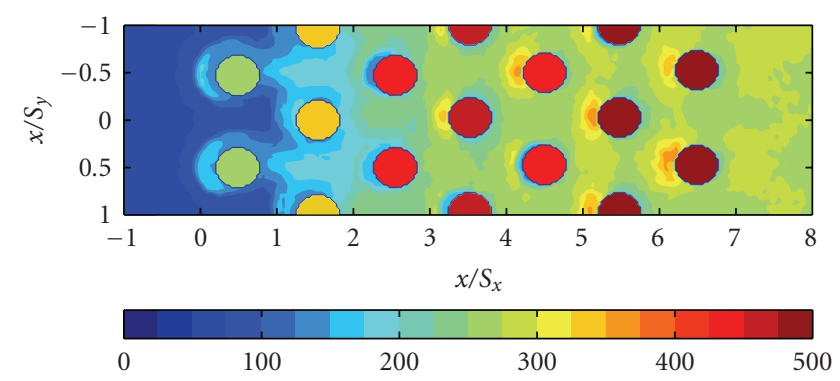

(a)

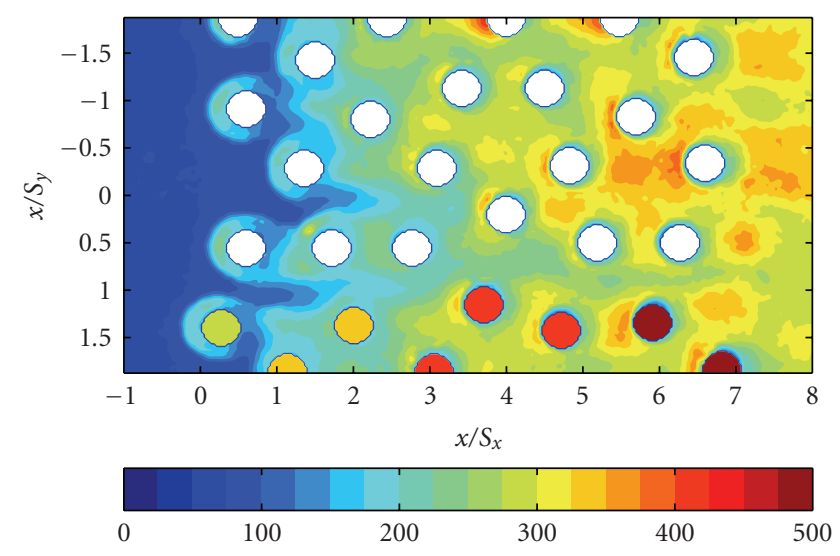

(b)

FIgURE 9: G2.5 and G2.6 endwall HTC $\left[\mathrm{W} / \mathrm{m}^{2} \mathrm{~K}\right]$ map- $\mathrm{Re}_{d 7}=$ 18000.

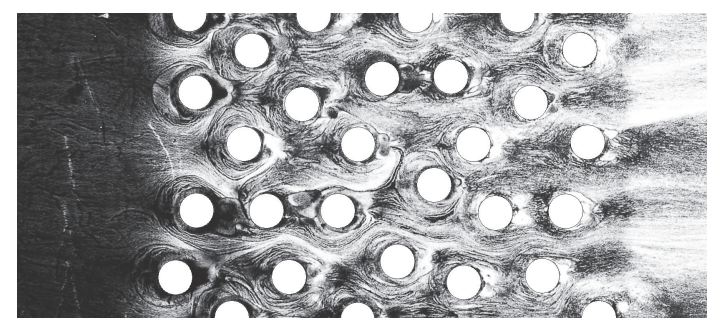

FIGURE 10: Surface flow visualization of G2.6.

over the pin fin surface with the inverse data reduction, in the G2.6 geometry, such measurement was performed in only 8 of the 29 pins that make up a repeatable array.

The two HTC maps in Figure 9 clearly show the different flowfield induced by the different pin fin arrangements . As expected, the HTC distribution is symmetric in the staggered configuration (G2.5), a stagnation region is present in front of each pin fin and the spanwise averaged HTC increases row by row. The pentagonal configuration shows a nonuniform development of the HTC map, actually there are noticeable differences in the spanwise direction. Due to pin fin distribution, in some areas they work as an inline array, while in other areas as staggered. For instance, for $y / S_{y}=0.9-1$, there are no pin fins over the endwall and then the HTC are lower. On the contrary, for $-1<y / S_{y}<0$, pin fins arrangement is similar to a staggered configuration and then heat transfer in that area is higher.

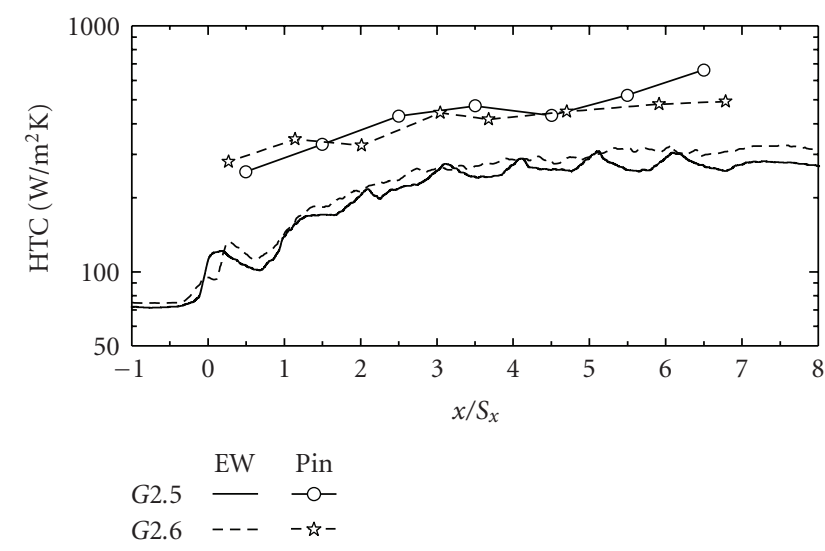

FIGURE 11: G2.5 and G2.6 endwall spanwise averaged and pin fin $\mathrm{HTC}-\mathrm{Re}_{d 7}=18000$.

The surface flow visualization in Figure 10 confirms the nonuniform flowfield. Anyway, the stagnation point and the recirculation ahead and behind each pin are almost always in line with the mainstream direction, while when two pin are in line, they are not clearly distinguishable. In this visualization, the low HTC area present at $y / S_{y}=$ $0.9-1$ is depicted by a single streamline that runs along the streamwise direction.

The spanwise-averaged endwall HTC values presented in Figure 11 show the heat transfer enhancement in the streamwise direction of both configurations. In the staggered array (G2.5), the stagnation ahead each pin fin row is visible, while for the pentagonal arrangement, it is noticeable only for $x / S_{x}=0.4$ where there are three aligned pin fins. Finally, looking at the spanwise-averaged endwall values, the G2.6 configuration always shows higher HTC values, especially for $x / S_{x}>5$. On the other hand, the pin fin HTC values are always slightly higher in the staggered configuration (G2.5), with the consequence that the overall heat transfer performance of both geometries is the same (Figure 12).

As for the elliptic configurations, in Figure 12, the overall Nusselt number of the $L 1$ region of both configurations is compared to the $L 0$ values. The entrance region values are, as expected, the same for both configurations, while it is surprising that the two pin fin arrangements generate an equal heat transfer enhancement too.

Some interesting considerations can be drawn looking at the row by row Nusselt values of the staggered configuration reported in Figure 13 (having a nonuniform arrangement, the definition of row by row values is not possible for the pentagonal array). Due to the higher number of rows, the aforesaid behavior for the spanwise elliptic geometry G2.2 is now more evident. The heat transfer capability of each row increases quickly up to the third row, then it is quite constant up to the fifth, increasing once again in the last two rows. Finally, the comparison with the correlation proposed by [4] for a constant height duct shows a general good agreement even if the flow acceleration due to the wedge-shaped duct leads to a lower dependence on the Reynold number, as was already reported once again by [4]. 


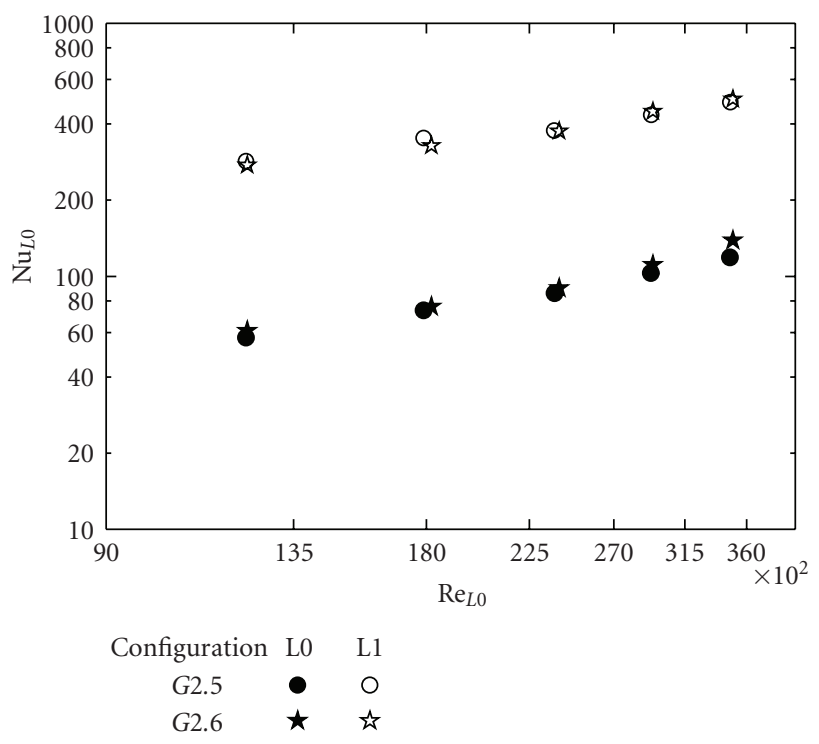

FIgURe 12: G2.5 and G2.6 data- $-\mathrm{Nu}_{L 0}$ versus $\mathrm{Re}_{L 0}$.

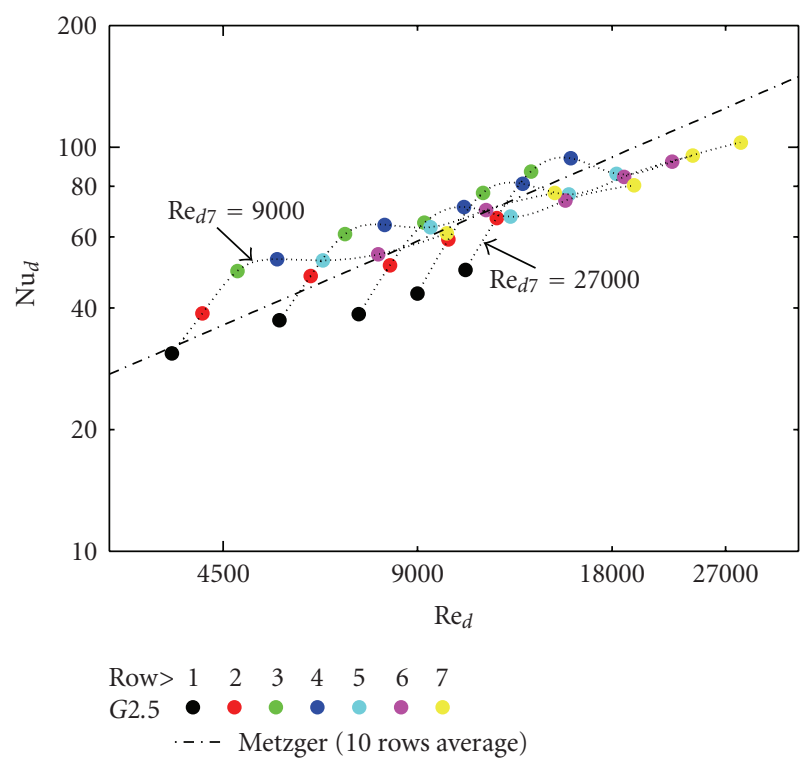

FiguRE 13: G2.5 row by row heat transfer data.

\subsection{Pressure losses}

In order to complete the experimental investigation, pressure losses of the four configurations were calculated using the definition of (4).

Due to the non-aerodynamic shape and to the very high flow velocity between the pin fins, the elliptic spanwise configuration G2.2 generates pressure losses up to seven times higher than the streamwise one. Such difference, also compared to the heat transfer enhancement capability that is only twice, demonstrates that the spanwise-oriented pin fins are not the optimal arrangement to be implemented in a trailing edge cooling system. Anyway, they can be employed in all the situations where low pressure losses are

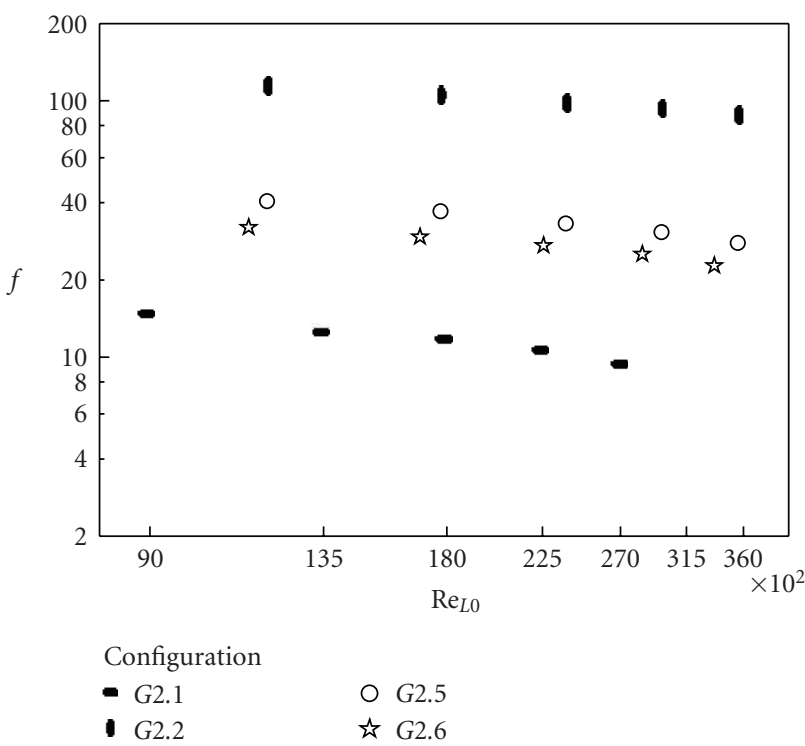

Figure 14: Pressure losses.

not required, while very high heat transfer coefficients are necessary.

Regarding the two circular configurations, the pentagonal arrangement G2.6 generates lower pressure losses than the standard staggered one. This result, considering that the two configurations showed the same heat transfer capabilities as well, proves the good capabilities of such innovative arrangement.

\section{CONCLUDING REMARKS}

The present study reports an experimental investigation of four different pin fin-based trailing edge cooling geometries. The heat transfer measurement were carried out employing a combined transient technique that allows to evaluate the separate contribution to heat transfer of pin fins and endwall surfaces. Results show that the pin fin surface heat transfer values are always higher than the surrounding endwall ones.

The comparison between streamwise- and spanwiseoriented pin fins with elliptic cross-section highlighted the very high capability in enhancing heat transfer of the spanwise array that is twice the streamwise one. On the other hand, pressure losses are seven times higher, making such array suitable when pressure losses are not a mandatory requirement of the cooling system. An oil and dye surface flow visualization allowed to depict the flowfield around each pin. Such investigation shows that pin fins of the first row have wider stagnation and recirculation areas compared to the following pins which exhibit a repeatable behavior.

An innovative array based on a pentagonal pin fin distribution has been then compared to a standard staggered configuration, keeping constant the overall pin fin density. Such pentagonal array shows a nonuniform heat transfer distribution over the endwall surface, anyway it seems to be very promising because it has the same performance of the 
standard staggered array in terms of heat transfer, while it generates lower pressure losses.

About the effects of the flow acceleration caused by the wedge-shaped duct, a general remark for all the four configuration can be done: the Nusselt number is less dependent on Reynolds number than in constant height ducts. Such behavior should be addressed to the turbulence intensity weakening typical of accelerating flows.

\section{Nomenclature}

A: $\quad$ Air passage area $\left(\mathrm{mm}^{2}\right)$

$D: \quad$ Circular pin fin diameter $(\mathrm{mm})$

$D: \quad$ Ellipse minor axes $(\mathrm{mm})$

Ma: Mach number

$N$ : Pin fin rows

Nu: Nusselt number

$H$ : Duct height

Re: Reynolds number

$S: \quad$ Spanwise pitch $(\mathrm{mm})$

$S_{x}: \quad$ Streamwise pitch $(\mathrm{mm})$

$L: \quad$ Wedge duct length $(\mathrm{mm})$

T: Temperature $\left({ }^{\circ} \mathrm{C}\right)$

c: $\quad$ Specific heat $(\mathrm{J} / \mathrm{kg} \mathrm{K})$

$f$ : Friction factor

$h$ : Heat transfer coefficient $\left(\mathrm{W} / \mathrm{m}^{2} \mathrm{~K}\right)$

$k$ : Thermal conductivity $(\mathrm{W} / \mathrm{mK})$

$\dot{m}:$ Air mass flow $(\mathrm{kg} / \mathrm{s})$

p: $\quad$ Pressure $(\mathrm{Pa})$

$r: \quad$ Fillet radius $(\mathrm{mm})$

$x$ : $\quad$ Streamwise coordinate $(\mathrm{mm})$

$y: \quad$ Spanmwise coordinate $(\mathrm{mm})$

$t: \quad$ Time (s)

$v: \quad$ Velocity $(\mathrm{m} / \mathrm{s})$

$\Delta p_{0}$ : Total pressure loss $(\mathrm{Pa})$

\section{Subscripts}

1-7: Pin fin row

L0: Entrance region

L1: Wedge region

L2 Exit region

PIN: Pin fin surface

EW: Endwall surface

d: Diameter

\section{Greeks}

$$
\rho: \text { Density }\left(\mathrm{kg} / \mathrm{m}^{3}\right) \text {. }
$$

\section{ACKNOWLEDGMENTS}

The reported work was performed within the European research project "Aerothermal Investigation of Turbine Endwalls and Blade (AITEB2), (RTD-Project 6th FP, Contract no. AST4-CT-2005-516113). The permission for the publication is gratefully acknowledged by the authors. Finally, the authors express their gratitude to Mr. Fabio Mantegna for his fundamental support in the experimental activities.

\section{REFERENCES}

[1] D. E. Metzger and S. W. Haley, "Heat transfer experiments and flow visualization for arrays of short pin fins," in Proceedings of the 27th International Gas Turbine Conference and Exhibit, p. 7, London, UK, January 1982, ASME paper 82-GT-138.

[2] D. E. Metzger, C. S. Fan, and S. W. Haley, "Effects of pin shape and array orientation on heat transfer and pressure loss in pin fin arrays," Journal of Engineering for Gas Turbines and Power, vol. 106, no. 1, pp. 252-257, 1984.

[3] G. J. Van Fossen, "Heat-transfer coefficients for staggered arrays of short pin fins," Journal of Engineering for Power, vol. 104, no. 2, pp. 268-274, 1982.

[4] D. E. Metzger, W. B. Shepard, and S. W. Haley, "Row resolved heat transfer variations in pin-fin arrays including effects of non-uniform arrays and flow convergence," in Proceedings of the International Gas Turbine Conference and Exhibit, p. 9, Duesseldorf, Germany, June 1986, ASME paper 86-GT-132.

[5] Z. Wang, P. T. Ireland, and T. V. Jones, "Detailed heat transfer coefficient measurements and thermal analysis at engine conditions of a pedestal with fillet radii," in Proceedings of the International Gas Turbine and Aeroengine Congress and Exposition, pp. 1-10, Cincinnati, Ohio, USA, May 1993, ASME paper 93-GT-329.

[6] Z. Wang, P. T. Ireland, T. V. Jones, and S. T. Kohler, "Measurements of local heat transfer coefficient over the full surface of a bank of pedestals with fillet radii," in Proceedings of the International Gas Turbine and Aeroengine Congress and Exposition, pp. 1-8, Hague, The Netherlands, June 1994, ASME paper 94-GT-307.

[7] J.-J. Hwang and C.-C. Lui, "Detailed heat transfer characteristic comparison in straight and 90-deg turned trapezoidal ducts with pin-fin arrays," International Journal of Heat and Mass Transfer, vol. 42, no. 21, pp. 4005-4016, 1999.

[8] J.-J. Hwang and C.-C. Lu, "Lateral-flow effect on endwall heat transfer and pressure drop in a pin-fin trapezoidal duct of various pin shapes," Journal of Turbomachinery, vol. 123, no. 1, pp. 133-139, 2001.

[9] J.-J. Hwang and C.-C. Lui, "Measurement of endwall heat transfer and pressure drop in a pin-fin wedge duct," International Journal of Heat and Mass Transfer, vol. 45, no. 4, pp. 877889, 2001.

[10] S. C. Arora and W. Abdel-Messeh, "Characteristics of partial length circular pin fins as heat transfer augmentors for airfoil internal cooling passages," Journal of Turbomachinery, vol. 112, no. 3, pp. 559-565, 1990.

[11] D. E. Metzger, R. A. Berry, and J. P. Bronson, "Developing heat transfer in rectangular ducts with staggered arrays of short pin fins," Journal of Heat Transfer, vol. 104, no. 4, pp. 700-706, 1982.

[12] M. K. Chyu, H. Ding, J. P. Downs, and F. O. Soechting, "Determination of local heat transfer coefficient based on bulk mean temperature using a transient liquid crystals technique," Experimental Thermal and Fluid Science, vol. 18, no. 2, pp. 142-149, 1998.

[13] M. K. Chyu, "Heat transfer and pressure drop for short pinfin arrays with pin-endwall fillet," Journal of Heat Transfer, vol. 112, no. 4, pp. 926-932, 1990.

[14] J. J. Hwang and C. C. Lui, "Lateral-flow effect on endwall heat transfer and pressure drop in a pin-fin trapezoidal duct of various pin shapes," in Proceedings of the ASME Turbo Expo, p. 9, Munich, Germany, May 2000, ASME paper 2000-GT-0232.

[15] B. Facchini, L. Innocenti, and L. Tarchi, "Pedestal and endwall contribution in heat transfer in thin wedge shaped trailing 
edge," in Proceedings of the ASME Turbo Expo: Power for Land, Sea \& Air, vol. 3, pp. 101-111, Vienna, Austria, June 2004, ASME paper GT2004-53152.

[16] S. P. Chen, P. W. Li, M. K. Chyu, F. J. Cunha, and W. AbdelMesseh, "Heat transfer in an airfoil trailing edge configuration with shaped pedestals mounted internal cooling channel and pressure side cutback," in Proceedings of the ASME Turbo Expo: Power for Land, Sea \& Air, pp. 819-828, Barcelona, Spain, May 2006, ASME paper GT2006-91019.

[17] P. T. Ireland, Z. Wang, and T. V. Jones, "Liquid crystal heat transfer measurements," in Measurements Techniques, Lecture Series 1993-05, von Karman Institute for Fluid Dynamics, Brussels, Belgium, 1993.

[18] C. Camci, "Liquid crystal thermography," in Temperature Measurements, Lecture Series 1996-09, von Karman Institute for Fluid Dynamics, Brussels, Belgium, 1996.

[19] P. T. Ireland and T. V. Jones, "Liquid crystal measurements of heat transfer and surface shear stress," Measurement Science and Technology, vol. 11, no. 7, pp. 969-986, 2000.

[20] M. K. Chyu, Y. C. Hsing, T. I.-P. Shih, and V. Natarajan, "Heat transfer contributions of pins and endwall in pin-fin arrays: effects of thermal boundary condition modeling," in Proceedings of the International Gas Turbine \& Aeroengine Congress \& Exhibition, p. 8, Stockholm, Sweden, June 1998, ASME paper 98-GT-175.

[21] ANSI/ASME, "Measurement uncertainty: part 1, instrument and apparatus," 1985, ANSI/ASME PTC 19.I-1985.

[22] S. J. Kline and F. A. McClintock, "Describing uncertainties in single sample experiments," Mechanical Engineering, vol. 75, pp. 3-8, 1953.

[23] O. Uzol and C. Camci, "Elliptical pin fins as an alternative to circular pin fins for gas turbine blade cooling applications part 1: endwall heat transfer and total pressure loss characteristics," in Proceedings of the ASME Turbo Expo International Gas Turbine Institute Conference, New Orleans, La, USA, June 2001, ASME paper 2001-GT-0180.

[24] O. Uzol and C. Camci, "Elliptical pin fins as an alternative to circular pin fins for gas turbine blade cooling applications part 2: wake flow field measurements and visualization using particle image velocimetry," in Proceedings of the ASME Turbo Expo International Gas Turbine Institute Conference, New Orleans, La, USA, June 2001, ASME paper 2001-GT-181. 

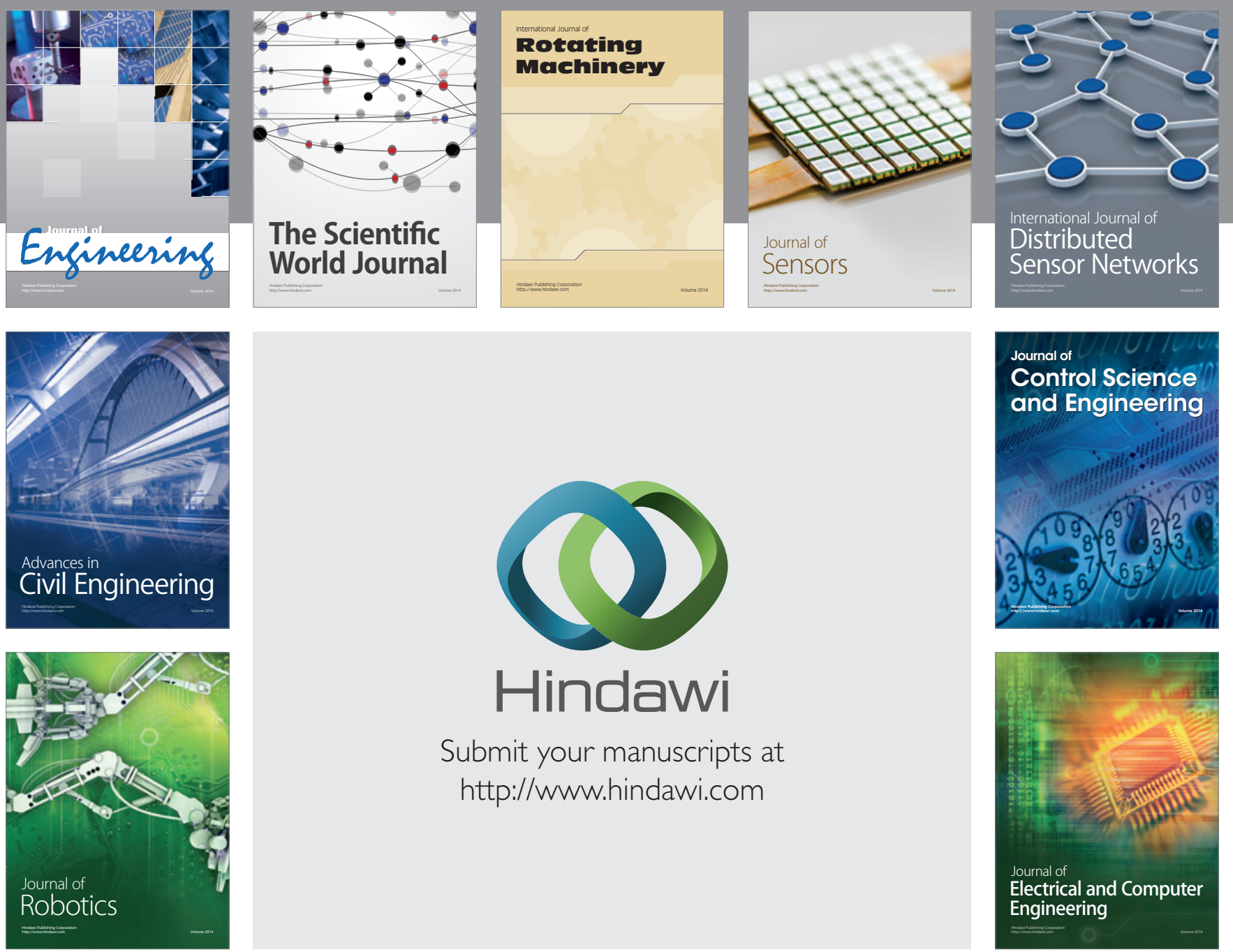

Submit your manuscripts at

http://www.hindawi.com
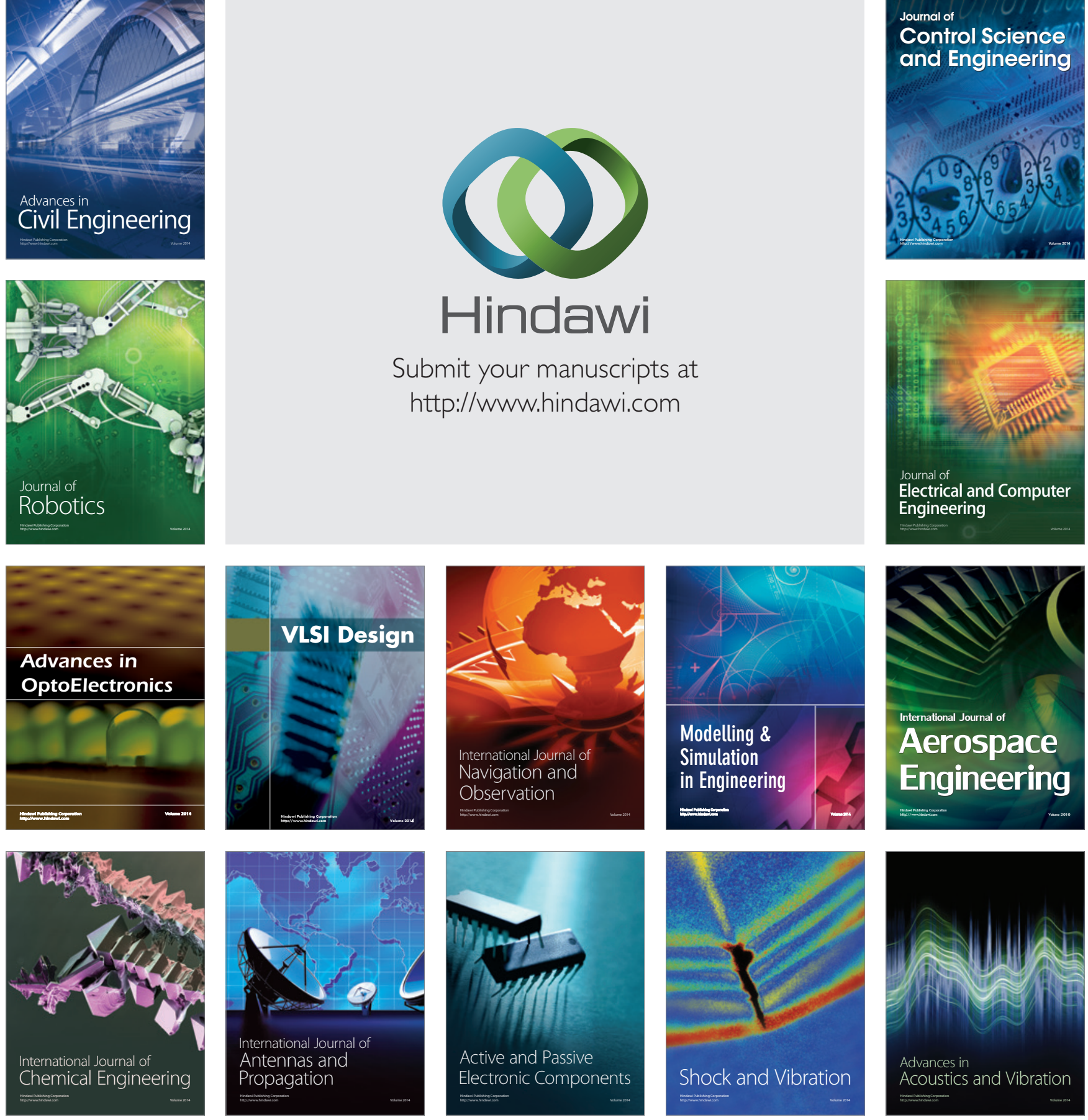